\title{
Prediction and verification of the single cladding layer thickness for laser solid forming
}

\author{
Jun-hua Wang ${ }^{1,2}$, Fu-zhu Han ${ }^{1,2,{ }^{*}}$ and Wei-sheng Ying ${ }^{1,2}$ \\ ${ }^{1}$ Department of Mechanical Engineering, Tsinghua University, Beijing 100084, China \\ 2 Beijing Key Lab of Precision/Ultra-precision Manufacturing Equipments and Control, Tsinghua University, Beijing 100084, China
}

\begin{abstract}
In order to accurately measure the powder effective utilization rate and to predict the single cladding layer thickness in laser solid forming process, a new method to measure powder distribution density is presented in this paper. Using this method, the powder material entering molten pool was collected by a copper tube with the same size as laser spot and its mass was measured to accurately calculate powder effective utilization rate in the laser solid forming process. And then, the single cladding layer thickness were calculated according to the conservation law of powder material mass before and after forming, and the correctness of calculating results was verified via a series of single track experiments. The results show that the theoretical calculation results of single cladding layer thickness are well coincident with the experimental results, and it proves that the measurement method of powder distribution density is accurate and reliable. In addition, laser power has a great influence on the geometry size of single cladding layer. Therefore, the influence of laser power on the geometry size of single cladding layer should be taken into account when establishing the prediction model of single cladding layer thickness.

Key words. Laser solid forming; Single cladding layer thickness; Powder effective utilization rate; Coaxial powder feeding; Powder concentration;
\end{abstract}

\section{Introduction}

Laser solid forming (LSF) is a new type of advanced manufacturing technology which can be used to rapidly fabricate the full dense complicated metallic components with high performance not using molds [1]. As to LSF technology, coaxial powder feeding technology is one of the most nuclear and key techniques [2]. In most cases, only a part of powder materials from powder feeder be sent to the molten pool and solidified into deposition materials because the size of powder flow is larger than that of laser spot. Therefore, it is necessary to explore powder effective utilization rate which is the ratio of the mass of powder materials entering the molten pool to that of powder materials from powder feeder per unit time [3]. Due to the mass of powder materials sent out by the powder feeder per unit time is known, whether the mass of powder materials entering the molten pool can be measured accurately has become the key to explore powder effective utilization rate.

Over the past decade, many researchers have studied powder flow distribution and its influences on the geometry size of single cladding layer. Typical method for powder flow distribution is based on optical methods. This method measure the intensity of the scattered light by the powder stream using a CCD sensor which provides the information to a digital image processing unit [4]. Due to the powder distribution has a positive correlation with the scattered light intensity, the powder distribution can be predicted according to the scattered light intensity. The main problem with optical methods is that they only obtain a qualitative concentration distribution [5]. This implies a great problem when the model is being validated, since it can only compare the powder flux distribution profiles without taking into account numerical values. Using this method, it is difficult to measure powder effective utilization rate accurately.

In order to accurately measure the powder effective utilization rate and to predict the single cladding layer thickness in laser solid forming process, this article proposes an alternative method to measure the mass of powder materials entering the molten pool. The methodology is relatively simple and is based on the weight measurement. Using this method, the powder material was collected by a copper tube with the same size as laser spot and its mass was measured to accurately calculate powder effective utilization rate. And then, the single cladding layer thickness were calculated according to the conservation law of powder material mass before and after forming, and the correctness of calculating results was verified via a series of single track experiments. The results show that the theoretical calculation results of single cladding layer thickness are well coincident with the experimental results.

\footnotetext{
*hanfuzhu@mail.tsinghua.edu.cn
} 


\section{Powder effective utilization rate}

\subsection{Powder distribution detection method}

Figure.1 shows the schematic diagram of the density distribution of powder flow. In Figure.1, there are significant differences in the size of powder flow and its distribution density in the horizontal plane with different spacing to the powder nozzles. Different powder flow size and distribution density will result in significant difference of powder effective utilization rate.
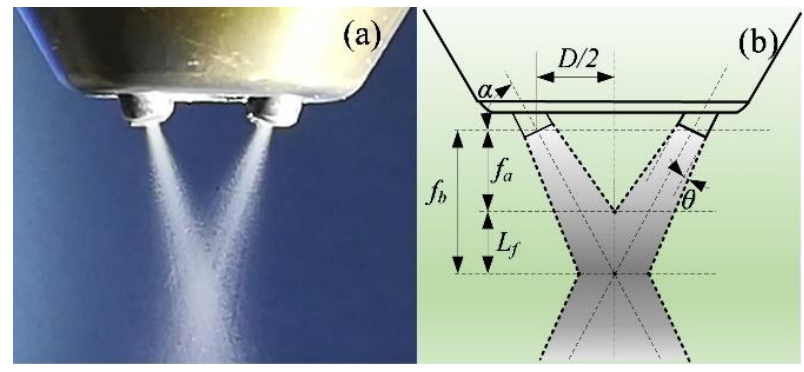

Figure 1. Schematic diagram of the density distribution of powder flow.

In order to accurately measure the powder effective utilization rate and to predict the single cladding layer thickness in LSF process, this article proposes a new detection method to measure the mass of powder materials entering the molten pool, its schematic diagram as shown in Figure.2(a). The methodology is relatively simple and is based on the weight measurement. Using this method, the powder material entering molten pool was collected by a copper tube with the same size as laser spot, and its mass was measured to accurately calculate the powder effective utilization rate. When the powder feeding system works, the powder particles falling into the test area freely fall into the powder container along the copper pipe. By measuring the mass of powder materials entering the container per unit time, the powder effective utilization rate in the test position can be calculated accurately. The distribution density of powder flow was determined by changing the test position in the horizontal plane with different spacing to the powder nozzles.

Using the method above, the distribution density in different horizontal planes were tested to clearly identify the distribution density of powder flow under coaxial powder feeding mode. The distance from the test horizontal planes to the nozzle was set to $11 \mathrm{~mm}, 12 \mathrm{~mm}$, $14.5 \mathrm{~mm}$ and $19 \mathrm{~mm}$, respectively. Measure specific time was set as $2 \mathrm{~min}$. In the experiments, the powder particles with the size of $75 \sim 185 \mu \mathrm{m}$ used in the experiments was martensitic stainless steel $\left(17-4 \mathrm{PH}\right.$, density $\left.=7.8 \mathrm{~g} / \mathrm{cm}^{3}\right)$. The schematic diagram of measurement process and the measurement results of powder distribution density in $\mathrm{X} / \mathrm{Y}$ axis directions of different horizontal planes are shown in Figure.2(b). As shown in Figure.2(b), there are significant differences in distribution density in $\mathrm{X} / \mathrm{Y}$ axis directions of different horizontal planes. When $\mathrm{H}=14.5$ $\mathrm{mm}\left(\mathrm{H}=f_{b}\right)$, the test horizontal plane is the theoretical focal plane of coaxial powder feeding system. In this horizontal plane, the distribution density of powder flow in $\mathrm{X} / \mathrm{Y}$ axis directions presents the Gaussian distribution feature, and the powder density reaches to the maximum. When $\mathrm{H}=11 \mathrm{~mm}$, the distribution density curves no longer obey the Gaussian distribution, but presents the double hump distribution characteristics.
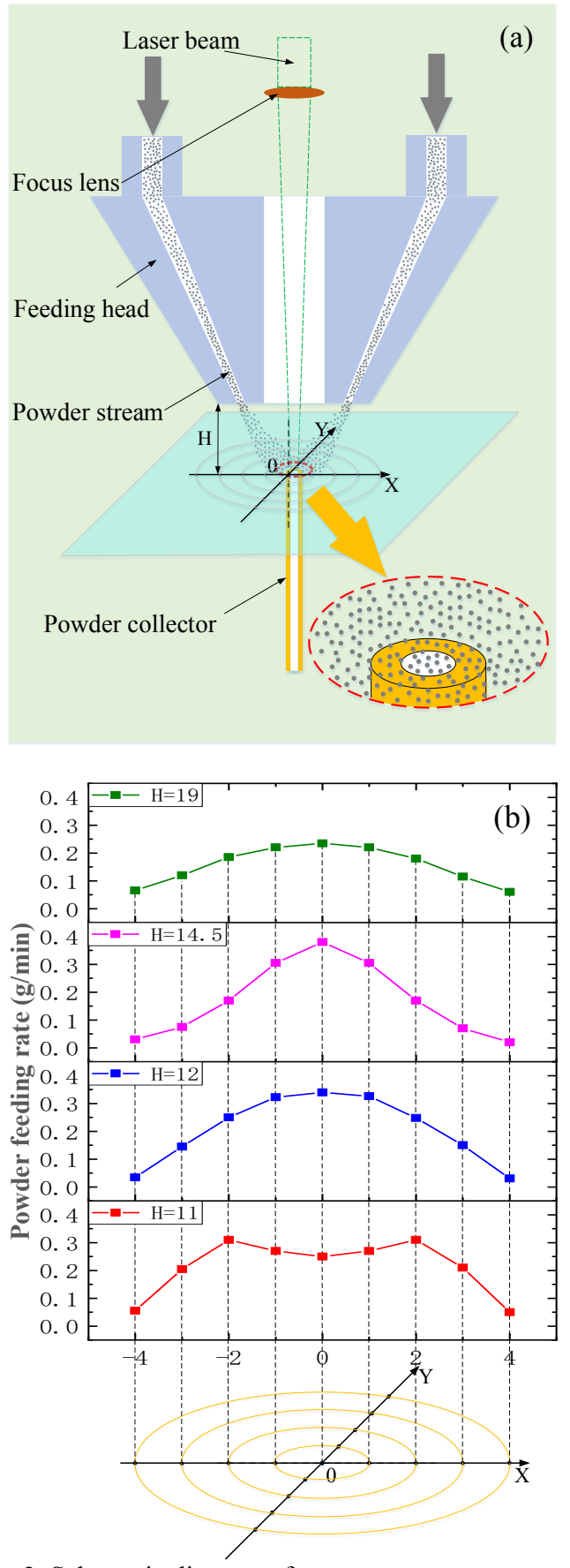

Figure 2. Schematic diagram of measurement process and the measurement results of powder distribution density in $\mathrm{X} / \mathrm{Y}$ axis directions of different horizontal planes.

\subsection{Measurements of powder effective utilization rate}

According to the detection results of distribution density of powder flow in $\mathrm{X} / \mathrm{Y}$ axis directions of different horizontal planes under the coaxial powder feeding mode, the powder distribution density varies significantly in the laser beam center in different horizontal planes. This will 
result in a significant difference in powder effective utilization rate.

The powder effective utilization rate varies in the laser beam center in different horizontal planes when the radius of laser beam is $0.5 \mathrm{~mm}$, as shown in Figure.3. In Figure.3, when $\mathrm{H}=14.5 \mathrm{~mm}\left(\mathrm{H}=f_{b}\right)$, the powder effective utilization rate reaches the maximum value, around $5.2 \%$. This is because the powder distribution density in the laser beam center is the largest at this time. Within the range of $\mathrm{H}<14.5 \mathrm{~mm}$, the powder effective utilization rate decreases rapidly with decreasing of $\mathrm{H}$ value. When $\mathrm{H}=7.5 \mathrm{~mm}$, the powder effective utilization rate is only $0.2 \%$. Meanwhile, within the range of $\mathrm{H}>14.5 \mathrm{~mm}$, the powder effective utilization rate decreased gradually with increasing of $\mathrm{H}$ value, but the reduction rate is significantly lower than that within the range of $\mathrm{H}<14.5 \mathrm{~mm}$. This is well coincident with the variation trend of powder distribution density at the laser beam center in different horizontal planes.

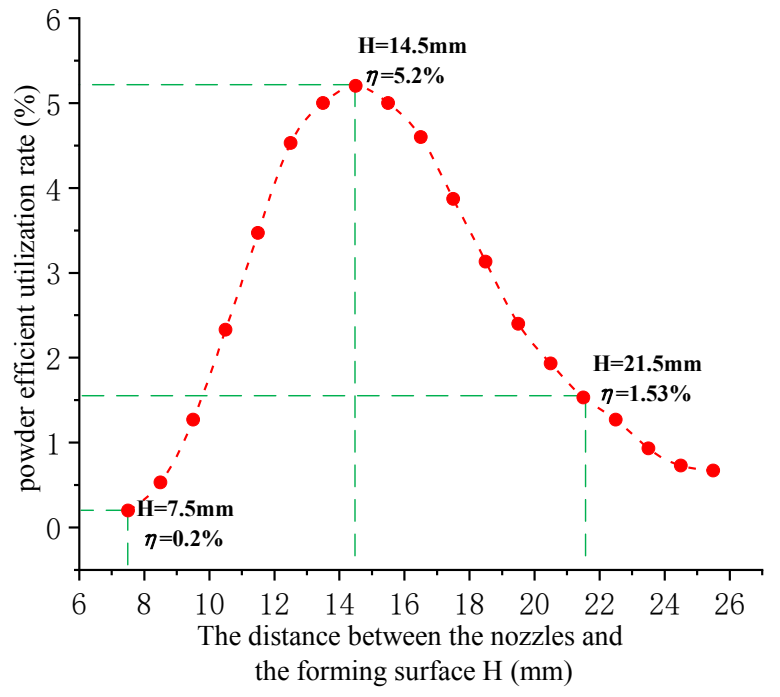

Figure 3. Powder effective utilization rate varies in the center of laser beam in different horizontal planes.

\section{Single cladding layer thickness}

\subsection{Prediction model}

In the actual LSF process, the powder materials from powder feeder be sent to the molten pool and solidified into deposition materials. According to the conservation law of powder material mass before and after forming, the single cladding layer thickness can be calculated. For simplicity, we assume that the shape and size of the molten pool are the same as that of the laser beam on the forming surface, and the cross section of the cladding layer is approximately rectangular rather than arched. Based on the measurements of powder effective utilization rate above, the computational formula of single cladding layer thickness can be obtained as follow:

$$
\Delta h=\frac{1000 M_{P} \eta}{60 \rho v B}
$$

where $M p$ is powder feeding rate $(\mathrm{g} / \mathrm{min}), \eta$ is powder effective utilization rate $(\%), \rho$ is powder density $\left(\mathrm{g} / \mathrm{cm}^{3}\right)$, $v$ is laser scanning speed $(\mathrm{mm} / \mathrm{s}), B$ is laser beam diameter (mm).

From the formula (1), we know that single cladding layer thickness is mainly determined by powder feeding rate, powder effective utilization rate, powder density, laser scanning speed, and the size of laser beam. Powder effective utilization rate and the size of laser beam are constant when the distance between the coaxial powderfeeding nozzles and the forming surface remains unchanged in the LSF process, single cladding layer thickness is mainly affected by powder feeding rate and scanning speed.

\subsection{Experimental detection}

In order to verify the accuracy of the prediction model of single cladding layer thickness, the focal plane at $\mathrm{H}=14.5 \mathrm{~mm}$ was set as the forming surface for the single track experiments. The powder materials and the parameters used in the experiments are listed in Table.1.

Table 1. The powder material and the parameters used in the experiments

\begin{tabular}{|c|c|}
\hline Material and Parameters & Values \\
\hline Powder material & $\begin{array}{c}\text { Martensite stainless } \\
\text { steel }(17-4 \mathrm{ph})\end{array}$ \\
\hline Powder density $\left(\mathrm{g} / \mathrm{cm}^{3}\right)$ & 7.8 \\
\hline Powder size $(\mu \mathrm{m})$ & $75 \sim 185$ \\
\hline Laser power $(\mathrm{W})$ & 200 \\
\hline Laser spot $(\mathrm{mm})$ & 1.0 \\
\hline \begin{tabular}{c} 
Scanning speed $(\mathrm{mm} / \mathrm{s})$ \\
\hline $\begin{array}{c}\text { Powder feeding rate } \\
(\mathrm{g} / \mathrm{min})\end{array}$
\end{tabular} & $2 \sim 12$ \\
\hline
\end{tabular}

When the powder feed rate changes, the comparison results of single cladding layer thickness between theoretical calculation and experimental verification are shown in Figure.4. The top view and three-dimensional diagram of the single cladding layer in the experiments under different powder feed rates are shown in the figure.5. The theoretical calculation results of single cladding layer thickness increased linearly and the experimental results of single cladding layer thickness increased approximately linearly with increasing of powder feed rate. The theoretical calculation results show good agreement with the experimental results. Moreover, it is also found that, the experimental measurement values are slightly lower than the theoretical calculation values. 


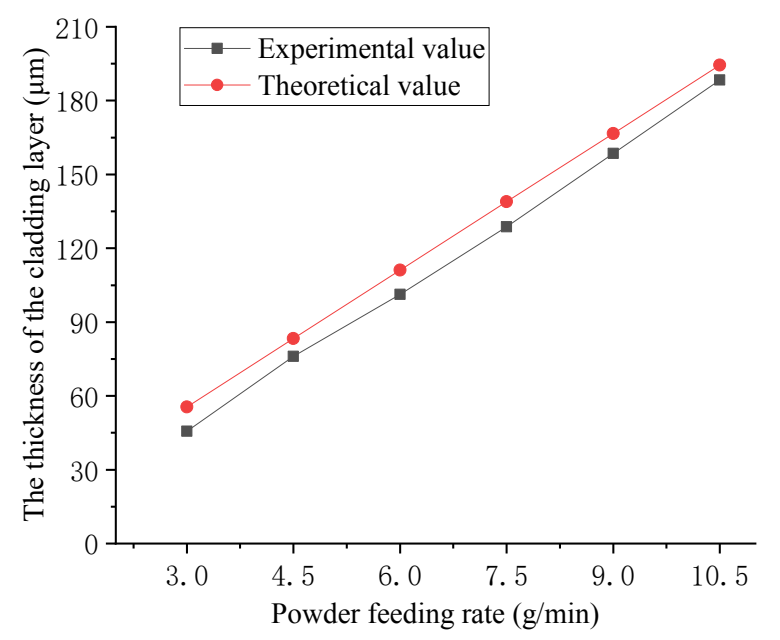

Figure 4. Comparison of the theoretical and experimental value of single cladding layer thickness under different powder feed rate.

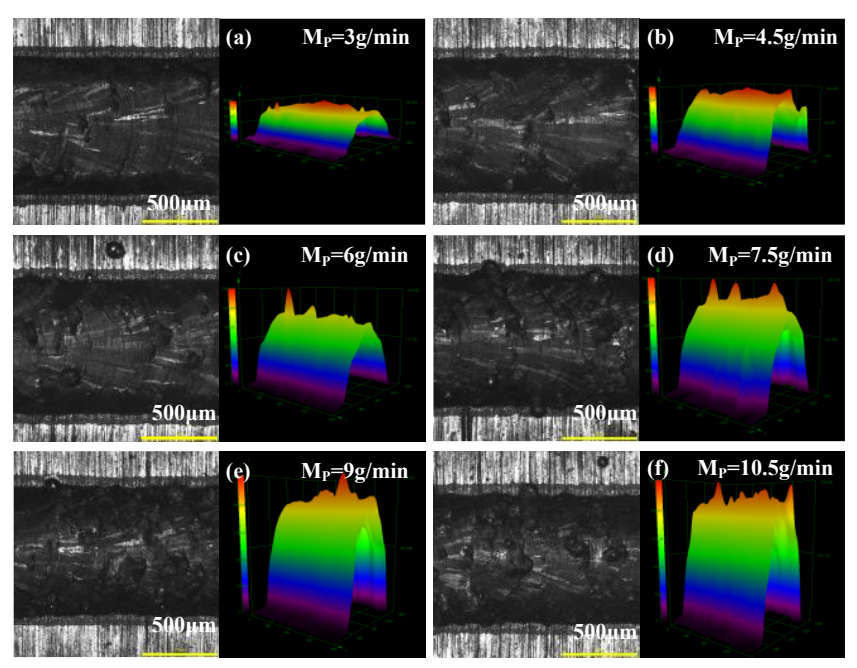

Figure.5 Comparison of single cladding layer morphology under different powder feed rates

When the scanning speed changes, the comparison results of single cladding layer thickness between theoretical calculation and experimental verification are shown in Figure.6. The top view and three-dimensional diagram of the single cladding layer in the experiments under different laser scanning speeds are shown in the figure.7. The theoretical calculation results and experimental results of single cladding layer thickness decreased with increasing of scanning speed. The reduction rate of single cladding layer thickness also decreased with increasing of scanning speed. Approximately inversely proportional relationship was established between the single cladding layer thickness and the scanning speed.

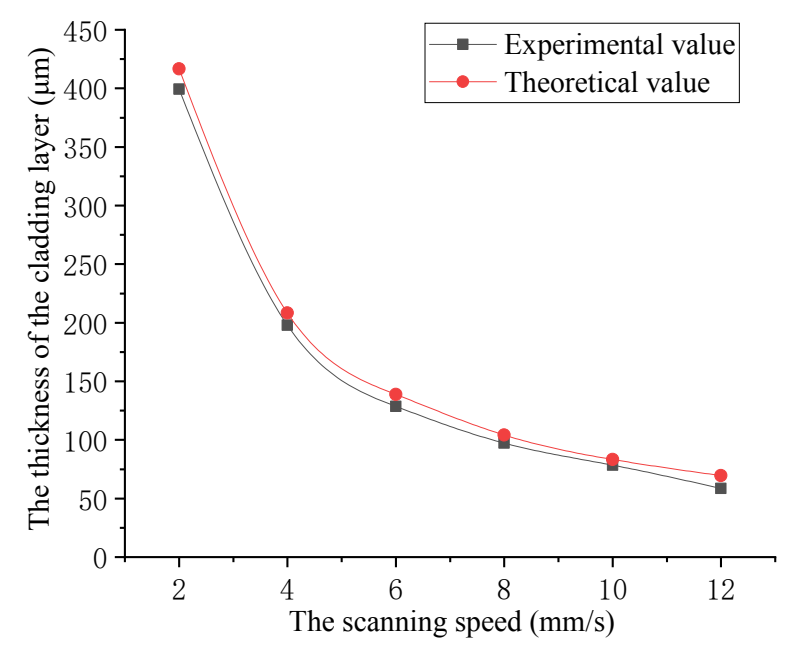

Figure 6. Comparison of the theoretical and experimental value of single cladding layer thickness under different scanning speed.

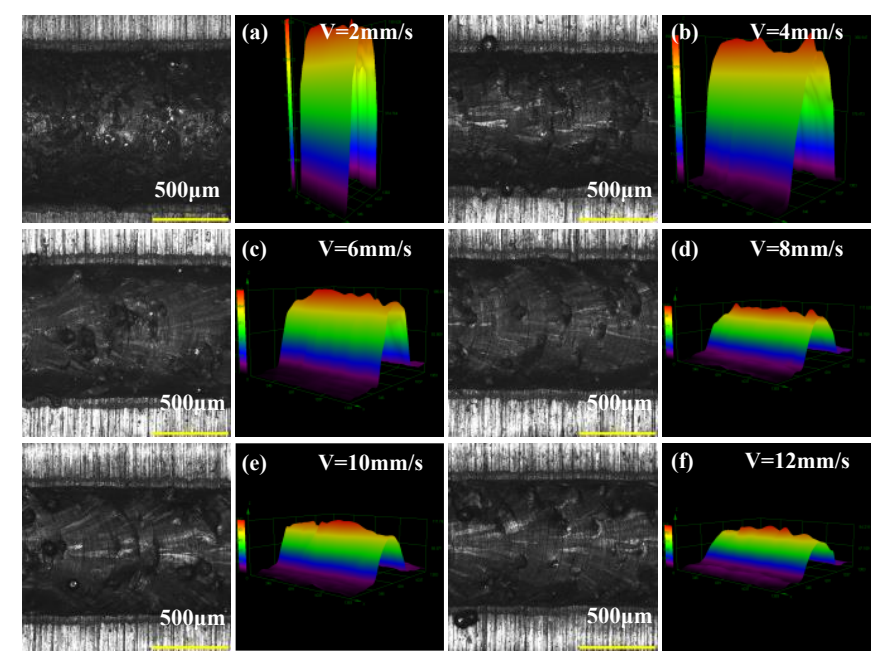

Figure.7 Comparison of single cladding layer morphology under different laser scanning speeds

\section{Analysis and discussion}

According to the results above, the theoretical calculation results of single cladding layer thickness are well coincident with the experimental results, and it proves that the measurement method of powder concentration is accurate and reliable. At the same time, it is also found that there are some subtle differences. The experimental measured results are all lower than the theoretical calculation results. By analysing experimental measured results, one possible reason is that the single cladding layer width is inconsistent with the laser spot diameter once the unsuitable laser power are used in the LSF process. In order to verify whether the above conjecture is true, this article investigated the influence of laser power on the width and thickness of single cladding layer via a series of single track experiments. The powder materials and the parameters used in the experiments are listed in Table. 2 . 
Table 2. The powder material and the parameters used in the experiments

\begin{tabular}{|c|c|}
\hline Material and Parameters & Values \\
\hline Powder material & $\begin{array}{c}\text { Martensite stainless } \\
\text { steel }(17-4 \mathrm{ph})\end{array}$ \\
\hline Powder density $\left(\mathrm{g} / \mathrm{cm}^{3}\right)$ & 7.8 \\
\hline Powder size $(\mu \mathrm{m})$ & $75 \sim 185$ \\
\hline Laser power $(\mathrm{W})$ & $140 \sim 360$ \\
\hline Laser spot $(\mathrm{mm})$ & 1.0 \\
\hline Scanning speed $(\mathrm{mm} / \mathrm{s})$ & 6 \\
\hline $\begin{array}{c}\text { Powder feeding rate } \\
(\mathrm{g} / \mathrm{min})\end{array}$ & 7.5 \\
\hline
\end{tabular}

The experimental results of the width and thickness of single cladding layer under different laser power are shown in Figure.8. As shown in Figure.8, the width and thickness of single cladding layer increase with increasing of laser power. The theoretical calculation value of single cladding layer thickness is $138.9 \mu \mathrm{m}$ under the process parameters selected in the experiments. When the laser power is $260 \mathrm{~W}$, the width and thickness of single cladding layer are $1002.6 \mu \mathrm{m}$ and $140.2 \mu \mathrm{m}$, respectively. This experimental results are highly consistent with the theoretical calculation value. But, the results under other laser power conditions are inconsistent with the theoretical value. Therefore, it can be seen that the accuracy of the theoretical calculation value of single cladding layer can be affected by the laser power. The influence of laser power on the geometry size should be taken into account to improve the prediction accuracy when establishing the prediction model of single cladding layer thickness.

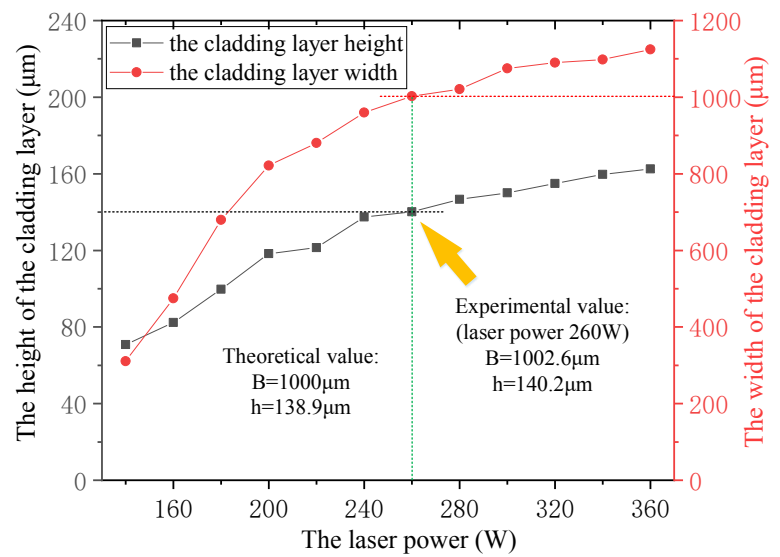

Figure 8. Experimental results of the width and thickness of single cladding layer under different laser power.

\section{Conclusions}

The main conclusions of this study are as follows:

1) The powder effective utilization rate has a significant impact on the geometry size of single cladding layer during LSF process. Due to the mass of powder materials sent out by the powder feeder per unit time is known, whether the mass of powder materials entering the molten pool can be measured accurately has become the key to measure powder effective utilization rate.

2) This work proposes an alternative method which is based on the weight measurement. Using this method, the powder material entering molten pool was collected by a copper tube with the same size as laser spot, and the correctness of calculating results was verified via a series of single track experiments.

3) The theoretical calculation results of single cladding layer thickness are well coincident with the experimental results, and it proves that the measurement method of powder distribution density is accurate and reliable.

4) Laser power has a great influence on the geometry size of single cladding layer. The influence of laser power on the geometry size should be taken into account when establishing the prediction model of single cladding layer thickness.

\section{Acknowledgements}

This work was supported by the National Key R\&D Program of China (2018YFB1105900) and the National Natural Science Foundation of China (51575308).

\section{References}

1. Tan H. Chen J. Zhang F. Lin X. and Huang W.D 2010 Optics \& Laser Technology. 42(1) 47-54.

2. Zhu G.X. Li D.C. Zhang A. and Tang Y. 2011 Optics \& Laser Technology. 43(1) 106-113.

3. Gong X.Y. Zhang Y.Z. and Liu M.K. 2013 International Journal of Advanced Manufacturing Technology. 67(9-12) 2501-2509.

4. Pinkerton and Andrew J. 2007 J. of Physics DApplied Physics. 40(23) 7323-7334.

5. Tabernero I. Lamikiz A. Ukar E. Lopez D. L. Angulo C. and Urbikain G. 2010 J. of Materials Processing Technology. 210(15) 2125-2134. 